\title{
Spatial Gradients in Particle Reinforced Polymers \\ Characterized by X-ray Attenuation and Laser Confocal Microscopy
}

\author{
R. Lagasse§ \& K. Thompson $\rrbracket$ \\ Organic Materials Department\$ \\ and Experimental Structural Mechanics Department $\mathbb{I}$ \\ Sandia National Laboratories \\ Albuquerque, NM 87185-1407
}

\begin{abstract}
The goal of this work is to develop techniques for measuring gradients in particle concentration within filled polymers, such as encapsulants. A high concentration of filler particles is added to such materials to tailor physical properties such as thermal expansion coefficient. Sedimentation and flow-induced migration of particles can produce concentration gradients that are most severe near material boundaries. Therefore, techniques for measuring local particle concentration should be accurate near boundaries. Particle gradients in an aluminafilled epoxy resin are measured with a spatial resolution of $0.2 \mathrm{~mm}$ using an $\mathrm{x}$-ray beam attenuation technique, but an artifact related to the finite diameter of the beam reduces accuracy near the specimen's edge. Local particle concentration near an edge can be measured more reliably using microscopy coupled with image analysis. This is illustrated by measuring concentration profiles of glass particles having $40 \mu \mathrm{m}$ median diameter using images acquired by a confocal laser fluorescence microscope. The mean of the measured profiles of volume fraction agrees to better than $3 \%$ with the expected value, and the shape of the profiles agrees qualitatively with simple theory for sedimentation of monodisperse particles. Extending this microscopy technique to smaller, micron-scale filler particles used in encapsulants for microelectronic devices is illustrated by measuring the local concentration of an epoxy resin containing 0.41 volume fraction of silica.
\end{abstract}

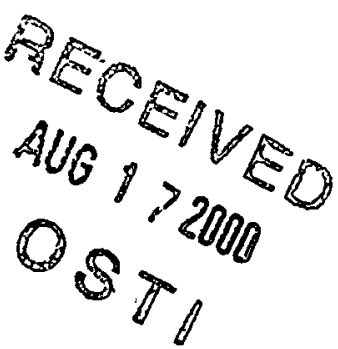




\section{DISCLAIMER}

This report was prepared as an account of work sponsored by an agency of the United States Government. Neither the United States Government nor any agency thereof, nor any of their employees, make any warranty, express or implied, or assumes any legal liability or responsibility for the accuracy, completeness, or usefulness of any information, apparatus, product, or process disclosed, or represents that its use would not infringe privately owned rights. Reference herein to any specific commercial product, process, or service by trade name, trademark, manufacturer, or otherwise does not necessarily constitute or imply its endorsement, recommendation, or favoring by the United States Government or any agency thereof. The views and opinions of authors expressed herein do not necessarily state or reflect those of the United States Government or any agency thereof. 


\section{DISCLAIMER}

Portions of this document may be illegible in electronic image products. Images are produced from the best available original document. 


\section{INTRODUCTION}

Many polymers used in engineering applications contain a high concentration of particulate modifiers. For example, polymer encapsulants for electronic devices are modified with $\sim 50 \mathrm{~V} \%$ of oxide particles, often silica, in order to reduce the thermal expansion coefficient I. One application having current interest is the Direct-Chip-Attachment packaging scheme in which an encapsulant fills the gap between chip and substrate. Spatially uniform distribution of particles is desired to achieve spatially uniform encapsulant properties, such as thermal expansion coefficient and elastic modulus. A uniform distribution of particles is also desired in order to simplify modeling the flow of these materials ${ }^{2}$. The present investigation is aimed at developing techniques for determining the spatial uniformity of particle concentration in highly filled polymer encapsulants.

One mechanism that can cause a spatial gradient in concentration and consequent nonuniform properties is sedimentation (or flotation) of particles differing in density from the fluid. Sedimentation in encapsulants can cause large changes in particle concentration over small distances, often near fluid boundaries. To clarify that statement, consider the simplest case of uniformly sized particles large enough that hydrodynamic forces dominate over interparticle forces, in a fluid having spatially uniform particle concentration, $\varphi_{0}$, as an initial condition. We consider the instructive, but unrealistic case (see Discussion) where the sedimentation velocity of all particles is the same and independent of local concentration. For this case, sedimentation will produce three zones of particle concentration ${ }^{3,4}$, as shown in Figure 1a. Here, a sediment having volume fraction $\varphi_{\max }$ forms at the lower fluid boundary. $\varphi_{\max }$ is the concentration where viscosity is so high that the suspension behaves like a solid (occurring at volume fraction 0.63 for random close packed monodisperse spheres ${ }^{5}$.) Figure la shows that sedimentation produces discontinuous steps in particle concentration at the two boundaries separating three different zones: the sediment, a middle zone having uniform concentration equal to the initial value, and a particle-free, clear zone. A simple mass balance relates the thickness of the sediment and the clear zone:

$$
t_{\text {sed }}=t_{\text {clear }} \frac{\varphi_{0}}{\varphi_{\max }-\varphi_{0}}
$$

As sedimentation proceeds, the thicknesses of the sediment and clear zone increase, as indicated by the dashed lines in Figure la.

In a more realistic case, where the sedimentation velocity depends on local particle concentration 3,4 and where the particles have a range of sizes (therefore, a range of velocities), the gradient in concentration at the boundaries will be very high instead of infinite, as illustrated in Figure 1b. At early stages of sedimentation, the two transition regions having very high concentration gradient will be close to the top and bottom boundaries of the fluid. This case of minimal sedimentation is relevant for polymer encapsulants since it is expected that technologically important materials will be formulated to mimimize the extent of sedimentation (in order to produce spatially uniform properties.) A technique measuring particle concentration profiles in an encapsulant must, therefore, be reliable near its boundaries.

Two additional mechanisms that can produce gradients in particle concentration, are

caused by flow deformation. Since these mechanisms are driven by hydrodynamic, not gravitational forces, they are operative even when the particles are neutrally buoyant. One 
mechanism is particle migration normal to the flow direction in a sheared suspension, from regions of high to low shear rate 6 . For the case of shearing flow through a channel, the region of highest shear rate, therefore the region of particle depletion, is near the channel wall. The second mechanism is accumulation of particles near an advancing free surface when a suspension flows into a cavity ${ }^{7}$. Both of these mechanisms can, like sedimentation, produce large gradients in particle concentration near boundaries.

The objective of the present work is to develop techniques for measuring spatial profiles in particle concentration in highly filled polymers over distances of order $100 \mu \mathrm{m}$, especially near boundaries. Most of the experiments utilize particles having diameters ranging from $1-100 \mu \mathrm{m}$. Extension to even smaller particles is considered near the end. In some cases, the polymer constituent contains particles of a second, immiscible polymer that must be distinguished from filler particles. Such immiscible elastomeric particles are used to mechanically toughen polymer encapsulants. The measurement techniques are validated using specimens that have experienced sedimentation (without significant flow), since sedimentation-derived concentration profiles can be predicted $^{3}, 4$, at least qualitatively.

\section{EXPERIMENTAL}

\section{A. Materials}

Important characteristics of the reinforcing particles are listed in Table 1. The distribution of diameters for each of the powders was measured via Mie analysis of angularly dependent scattered light (using an LS230 instrument from Coulter Corp.) In the table, $\mathrm{D}_{50}$ is the median value of the volume distribution of particle sizes, representing the diameter at which half of the total volume of particles is smaller and half larger. The $\mathrm{D}_{10}$ and $\mathrm{D}_{90}$ values have corresponding definitions. DSV is a different kind of average diameter, defined below. The alumina was a ground tabular product having irregular particle shape and a broad size distribution, indicated by the large spread between the $\mathrm{D}_{10}$ and $\mathrm{D}_{90}$ values in Table I. Three kinds of glass powders were employed, all having nominally spherical shape. One consisted of solid A-glass particles and the other consisted of hollow borosilicate glass particles having similar sizes. The third was a smaller size silica powder of the kind used in encapsulants for microelectronic devices.

The alumina was dispersed in two epoxy resins differing in rate of cure and, therefore, permitting a greater or lesser extent of particle sedimentation. The slower curing resin consisted of a liquid resin (di-glycidyl ether of bisphenol A type, Shell Epon 828) cured with a mixture of aromatic amines (Curing Agent $Z$ produced by Air Products and Chemicals). The curing kinetics of this epoxy have been characterized ${ }^{8}$. The faster curing resin consisted of a slightly lower molecular weight version of the same resin (Epon 826) cured with a mixture of two amines, a cycloaliphatic type (Ancamine 2049, produced by Air Products and Chemicals) and an aliphatic ether type (Jeffamine D-230, produced by Huntsman Petrochemical).

The glass and silica particles were dispersed in two different epoxy resins. One consisted

of the same Epon 828 cured with diethanol amine, whose curing kinetics are documented ${ }^{8}$. The second epoxy was the same except that it contained $9 \mathrm{wt} . \%$ of an elastomeric butadieneacrylonitrile copolymer modifier often added to improve mechanical toughness of epoxies, CTBN $1300 \times 88^{9}$. Both of these resins contained a very low concentration $(<0.01 \mathrm{wt}$ \%) of a fluorescent dye, Rhodamine-B. This was added to permit imaging the particles by laser confocal microscopy. 


\section{B. Procedures}

Specimens for x-ray radiography measurements were $6 \mathrm{~mm}$, parallel-faced slabs cut in a vertical orientation from larger castings. For confocal microscopy experiments, pieces of dyed, glass-filled epoxy from castings were mounted then polished using standard metallographic procedures. Unpolished specimens of the hollow-glass-filled epoxy produced poor quality micrographs (even though other polymer composites are said to require no special preparation for confocal microscopy ${ }^{10}$.)

$\mathrm{X}$-ray attenuation measurements employed a divergent beam, polychromatic $\mathrm{X}$-ray

source. The $\mathrm{x}$-ray tube had a tungsten anode operated at $140 \mathrm{kV}$ with a focal spot size of $0.2 \mathrm{x}$ $0.2 \mathrm{~mm}$. The beam was filtered by $1 \mathrm{~mm}$ beryllium and $16 \mathrm{~mm}$ aluminum sheets. Collimators on the $\mathrm{x}$-ray source and detector produced an effective direct-beam-illuminated spot on the specimen having dimension between 0.2 and $0.25 \mathrm{~mm}$ along the direction plotted in the figures to follow. In other words, measurements of $\mathrm{x}$-ray attenuation were obtained with spatial resolution of 0.2 to $0.25 \mathrm{~mm}$.

The laser confocal microscopy was performed using a Bio-Rad MRC-600 laser confocal scanning head mounted on a Nikon Diaphot inverted microscope. The specimen was illuminated with $568 \mathrm{~nm}$ light produced by a Krypton-Argon ion laser and a narrow pass filter. This nearly monochromatic excitation light was excluded from the image-forming mechanism by a long-pass $585 \mathrm{~nm}$ filter. Therefore, only light from fluorescence of the Rhodamine-B dye was utilized to produce the images. (Emission for this dye in methanol solution occurs as a broad peak centered at $565 \mathrm{~nm}$.) A 40x, 1.0 numerical aperture oil-immersion Nikon objective was fitted on the microscope and produced images having lateral dimensions of 420 by $320 \mu \mathrm{m}$. A variable aperture on the confocal head was adjusted to produce an optical section thickness of $2 \mu \mathrm{m}^{11}$. Specimens were translated precisely under the microscope using a micrometer driven, homemade jig. More discussion of confocal laser scanning microscopy is available ${ }^{12,13}$.

Scanning electron microscopy backscatter images of the silica-filled epoxy were obtained from a polished surface using a Hitachi S- 4500 microscope operating at $15 \mathrm{kV}$ accelerating voltage. Prior to the microscopy, the surface of each sample had been sputter coated with goldpalladium.

\section{DATA ANALYSIS}

- Measurements of fractional $\mathrm{x}$-ray transmission through alumina filled epoxy specimens were reduced to filler concentration using calibration specimens having known concentration, and the analysis summarized below. The fractional transmission $T$ of polychromatic radiation passing through a material consisting of two phases $\mathrm{A}$ and $\mathrm{B}$ (particles and epoxy) is given by

$$
\ln (T)=-\overline{\mu_{A}} t_{A}-\overline{\mu_{B}} t_{B}
$$

where $\bar{\mu}$ is the linear attenuation coefficient averaged over the spectrum of the radiation and $\mathrm{t}$ is the thickness of a phase. Issues involving averaging over the x-ray spectrum (beam-hardening complications) are accounted for by selecting calibration specimens having nearly the same composition and thickness as the test specimen. If phase $B$ attenuates the radiation much less than $\mathrm{A}$, Equation 2 reduces to

$$
\ln (T)=-\overline{\mu_{A}} t_{A}+K_{1}
$$

where $K_{1}$ is a constant that has smaller magnitude than either of the other two terms. This equation is a reasonable approximation for epoxy and alumina because for $140 \mathrm{keV} \mathrm{x}$ rays, $\bar{\mu}$ for epoxy is 3 times smaller than that for alumina and because the thicknesses of the two phases are 
similar (as will be seen below). Expressing the thickness of phase $A$ in terms of its volume fraction using a standard stereological relationship ${ }^{14}$ and rearranging permits the last equation to be expressed as

$$
\varphi_{A}=-\bar{\mu}_{A}^{-1} t^{-1} \ln (T)+t^{-1} K_{2}
$$

where $\varphi_{A}$ is the volume fraction of phase $A, t$ is the specimen thickness, and $K_{2}$ is a new constant equal to ${\overline{\mu_{A}}}^{-1} K_{1}=\left(-\overline{\mu_{B}} / \overline{\mu_{A}}\right) t_{B}$

The two unknowns in Equation $4, \bar{\mu}_{A}^{-1}$ and $\mathrm{K}_{2}$, were determined from a plot of $\mathrm{T}$ measurements on six calibration specimens, each having known thickness $t$ and alumina volume fraction $\varphi_{A}$. The plot followed the linear form of Equation 4 , confirming that $\bar{\mu}_{A}^{-1}$ and $\mathrm{K}_{2}$ were indeed constants. Equation 4 could then be used to determine spatial profiles of alumina volume fraction in a test specimen having known thickness by measuring profiles of the $\mathrm{x}$-ray fractional transmission $T$.

Regarding determination of local particle concentration by microscopy, it is well known that this can be derived from the area fraction of particles in an image ${ }^{14}$. Specifically, the volume fraction of particles is equal their area fraction on an infinitely thin plane section, as long as the section is representative of the material. If the section thickness is finite, but less than the particle size, the volume fraction $\varphi$ is related to the area fraction $A_{A}$ as follows.

$$
\varphi=A_{A}-1.5 h / D_{S V}
$$

where $h$ is the section thickness and DSV is the surface-volume average diameter of the particles $^{15}$, defined as $\sum_{i}\left(n_{i} D_{i}^{3}\right) /\left(n_{i} D_{i}^{2}\right)$, where $n_{\mathrm{i}}$ represents number fraction. DSV derived from size distribution measurements on the solid and glass particles is listed in Table 1. Subjectivity involved with determining the area fraction of particles $\mathrm{A}_{\mathrm{A}}$ from micrographs was minimized by employing an operator-independent method for segmenting the total area between particles and epoxy. This segmentation utilized an algorithm that performs a statistical t-test on an image brightness histogram. The segmentation algorithm implemented in commercial image analysis software, Image Pro Plus (from Media Cybernetics, LP), was employed in this work.

\section{RESULTS}

\section{A. X-ray attenuation measurements}

Figures 2 (a) and (b) show measurements of volume fraction of the alumina particles near the bottom surface of two epoxy specimens. The plots in (a) and (b) show results for the same alumina in a slower and a faster curing epoxy resin, respectively. The transformation from liquid to solid gel occurred after 7.8 hours vs. 6.7 hours of curing for the slower and faster curing epoxies. This gel transformation arrests sedimentation of alumina particles. In both plots, increasing abscissa values correspond to increasing distance from the bottom, but the absolute values of the abscissa have no significance.

The profile in Figure 2(a) shows, far from the bottom (at large distance values), a wide region of uniform concentration averaging 0.437 particle volume fraction. That value differs insignificantly (only 1\%) from the volume fraction calculated from the weights and densities of the epoxy's constituents, confirming the accuracy of the volume fraction measurements. The (a) profile also exhibits, close to the bottom, a $0.2 \mathrm{~mm}$ layer having higher than average particle concentration. The highest volume fraction measured in that layer is $12 \%$ above that in the 
uniform region. The thickness of the sedimented layer is only $0.2 \%$ of the total height of the specimen. (Only a very small part of the total specimen height is shown in Figure 2.) Continuing to still lower abscissa values, the profile exhibits a rapid drop in particle concentration, most likely caused by the finite-width $x$-ray beam straddling the bottom surface of the specimen. In principle, improving the spatial resolution of the particle concentration measurements would improve the accuracy of the concentration profile and probably increase the maximum value of particle concentration at the specimen bottom. Toward this end, we attempted to measure ${ }^{16}$ concentration profiles in alumina filled epoxy materials using a tomographic technique that utilized a synchrotron radiation source (X-ray Tomographic Microscopy ${ }^{17}$ ). This technique was capable of producing profiles having higher spatial resolution, on the order of $10 \mu \mathrm{m}$. These results will not be presented here because they still contained an edge-related artifact, showing that improving spatial resolution does not necessarily solve that problem.

The profile in Figure 2(b) conforms to the expectation that the same particles should exhibit less particle sedimentation in the faster curing epoxy than the slower curing material. In fact, no sedimentation can be detected in Figure 2(b). The drop in volume fraction near the bottom surface is again seen, confirming that it is a measurement artifact unrelated to the concentration profile.

A final comment on Figure 2(a) concerns the expected effect of the edge artifact for measurements near the top surface of a material containing sedimented particles. Sedimentation would cause a depletion in particle concentration near the top surface. The edge artifact would also cause a reduction near the top surface, as it does near the bottom. Extracting an accurate, artifact-free concentration profile near the top would be even more difficult than at the bottom (Figure 2(a)), because at the top, both sedimentation and the edge artifact would cause the concentration to change in the same direction.

\section{B. Microscopy images for determining local particle concentration}

To determine particle concentration profiles having better spatial resolution and fewer edge-related artifacts than the radiography results, microscopy coupled with image analysis for determining particle area fractions were employed. The main advantage of microscopy over beam attenuation methods is that the particle microstructure can be determined close to.a specimen edge, as long as that edge and the particle outlines can be unambiguously imaged. A disadvantage of microscopy is that care must be taken to image a statistically representative number of particles at each location within a specimen. The acceptably low scatter in measured particle concentration profiles to be shown below demonstrate that this requirement was satisfied in this work. To simplify the technique development, we initially used optical microscopy. This could not resolve the smallest particles in the alumina size distribution nor the silica particles used in this work, but it could resolve the solid and hollow glass particles.

Simple, reflected light optical microscopy imaged particle outlines very well for the solid glass particles dispersed in epoxy, but imaged poorly the hollow glass particles. The poor definition of hollow particle outlines in reflection micrographs is illustrated in Figure 3(a). Figure 3(b), however, shows that the fluorescence laser confocal images delineated the hollow particle outlines unambiguously. This excellent contrast between the epoxy phase and hollow particles in the confocal images is caused by the image being formed exclusively by light from the fluorescing Rhodamine- $\mathrm{B}$ dye. This dye is present only in the epoxy phase. The glass walls and hollow interiors of the particle contain no dye and so appear black, contrasting strongly with the bright epoxy. The confocal micrographs imaged the particle outlines for the solid glass particles equally well. Another desirable attribute of confocal microscopy is that the depth of the 
sampling volume within which particle outlines are imaged is small relative to the particle diameters and is known reliably. For the microscope configuration employed, this specimen depth had been determined previously to be $2 \mu \mathrm{m}^{\prime \prime}$. Confocal fluorescence microscopy was therefore employed for measuring concentration profiles for both types of glass particles.

\section{Concentration profiles from confocal micrographs}

Confocal microscopy via its "optical slicing" feature 12,13 has the capability to characterize material microstructure below the surface of a specimen . Nevertheless, in this work, particle volume fractions were determined from micrographs obtained very close to the specimen surface. The reason is explained using Figure 4. This shows a plot of volume fractions measured at different depths below the polished specimen surface. The plot for the solid glass particles exhibits $\pm 0.03-0.04$ variation in volume fraction with no monotonic trend with increasing depth below the surface. This variation is believed to be caused primarily by experimental scatter in measuring concentration of these particles, because similar scatter is seen in all measurements on this material (see next figure.) In contrast, the hollow particle results in Figure 4 show a monotonic increase in concentration at depths greater than 5 microns. This is believed to be caused by an artifact arising from attenuation of fluorescence produced by the dyed epoxy at those depths. Attenuation of light coming from the epoxy would make the dark, non-fluorescing particles appear to have higher concentration. A likely mechanism for attenuation is reflection of the light at the interior walls of the hollow particles. Figure 4 suggests that this attenuation is less significant in the material filled with the solid particles. It is therefore believed that the concentration measurements are accurate at depths less than $25 \mu \mathrm{m}$ for the solid particles and less than $5 \mu \mathrm{m}$ for the hollow particles. Concentration measurements shown below for both kinds of particles were performed at depths less than $5 \mu \mathrm{m}$.

Figure 5 is a plot of particle concentration vs. height for an epoxy filled with 0.49 volume fraction of solid glass particles. Sedimentation of the particles prior to the epoxy's gel point produced an excess near the bottom surface (at abscissa $=0$ ) and a depletion near the top surface. Those two zones at the specimen boundaries are separated by a middle zone of essentially constant concentration. The measured profile in Figure 5 is consistent with the schematic profile shown in Figure 1(b). The average concentration of glass particles over the middle zone agrees to within $0.2 \%$ with the volume fraction computed from the weights and densities of constituents in the filled epoxy (0.49.) The measured concentration in the middle zone, therefore, agrees with the initial, uniform concentration of particles before sedimentation started, again in agreement with Figure 1(b). The apparent asymmetry in the profile, i.e., the more gradual transition from the sedimented zone to the middle zone and the more abrupt transition from the middle zone to the depleted zone, is believed to be a real phenomenon that can be predicted from a model of particle sedimentation. That prediction will be explained in the Discussion. It should be noted that the results in the previous Figure 4 were obtained in the middle zone. It should also be noted that the volume fraction results in Figure 5 do not include the correction suggested by Equation 5 . Including that correction would have decreased the measured particle volume fraction in the middle zone to 0.41 , raising the discrepancy with the computed volume fraction to $16 \%$. The applicability of the correction for finite section thickness to these confocal micrographs is discussed below.

Figure 6 shows profiles for two epoxies containing different concentrations of the hollow glass particles. Flotation of these particles produced a depleted zone near the specimen bottom (at abscissa $=0$ ), a middle zone of essentially uniform concentration, and concentrated zone near the top. In the epoxy having higher average concentration, the middle zone particle volume 
fraction agrees with the value computed from weights and densities to within $2 \%$, while in the lower concentration material, those two values agree to better than $3 \%$. Note that an asymmetry like that shown previously in Figure 5 appears to be present in both profiles. That is, both profiles suggest that the transition region between the depleted zone and the middle zone is more abrupt than the transition between the middle zone and the concentrated zone. (That asymmetry is somewhat obscured in the higher concentration epoxy by the scatter in measured concentration values near both surfaces.) As in Figure 5, the volume fractions in Figure 6 do not include the correction for finite section thickness, because that would have worsened the agreement with the known particle concentration in the middle zone.

Many particle reinforced epoxy materials contain elastomeric toughening agents that form a separate population of spherical polymeric particles ${ }^{18}$. The confocal microscopy technique was able to distinguish elastomeric particles in order to determine the concentration of the glass filler particles alone. Figure 7 shows a confocal micrograph of an epoxy containing CTBN elastomeric modifier and 0.48 volume fraction of the hollow glass particles. The epoxy materials shown in this and the previous Figure 3(b) are the same except for the CTBN modifier particles. In Figure 7, the elastomeric particles can be distinguished because they are brighter than the glass particles. The brighter appearance of the elastomeric particles is most likely caused by their containing more fluorescent dye than the glass particles, which contain none. Measurements on 4 images obtained far from specimen boundaries produced an average, glass particle volume fraction (uncorrected by Equation 5) of $0.496 \pm 0.038$, which differs by only $3 \%$ from the value computed from the weights and densities of constituents.

We have made use of the confocal microscopy technique for measuring local filler concentration in our laboratory in connection with dielectric breakdown measurements on epoxy materials filled with the hollow glass particles. We needed to determine the particle concentration in a very small volume through which the breakdown propagated, because the breakdown strength is sensitive to the concentration of hollow particles. The dimensions of that material volume are shown schematically in Figure 8. The breakdown propagated through 0.5 $\mathrm{mm}$ layer of epoxy separating two $12 \mathrm{~mm}$ diameter spherical electrodes. We determined a particle volume fraction of 0.445 (with standard deviation of 0.037 ) in the region close to the center-line axis of symmetry between the electrodes (a region having boundaries $300 \mu \mathrm{m}$ above and below that axis.) The standard deviation was larger than normal because that region, having a minimum width of only about 12 average particle diameters, contained a relatively small number of particles. The concentration within the gap region was not significantly different from the average particle volume fraction of 0.487 . However, we did detect non-uniform particle concentration near electrode-epoxy interfaces that had a component of particle flotation velocity oriented along a normal to the interface. Particle depletion near an interface is shown in Figure 9(a), where the normal component of flotation velocity vector was directed away from the interface. Accumulation of particles is observed in Figure 9(b), where the normal component of flotation was directed toward the interface, as expected. It was not possible to measure with any accuracy the particle concentration in those two thin layers because they contained a statistically insignificant number of particles. We have used qualitative characterizations of particle concentration profiles like those shown in Figure 9 (a) and (b) and quantitative profiles like those shown in Figures 5 and 6 to determine the effect of different temperature schedules for curing filled epoxies on particle flotation or sedimentation. 


\section{Local concentration measurements for smaller particles}

Filler particles in encapsulants for packaging microelectronics are smaller than the particles used in the preceding experiments. The goal of this portion of the work was to demonstrate feasibility for determining microscopically the local concentration of those smaller particles. The silica particles listed in Table I were selected to represent fillers used in microelectronic encapsulants. Since an appreciable fraction of these particles was smaller than 1 micron, optical microscopy had to be replaced by electron microscopy. Analysis of backscattered scanning electron micrographs (taken far from specimen boundaries) of the silicaepoxy produced 0.40 volume fraction of particles. This agreed to within $2 \%$ with the volume fraction computed from weights and densities of the constituents. A typical micrograph of this material is shown in Figure 10. Analysis of electron micrographs therefore shows promise for determining profiles of particle concentration in microelectronic encapsulants.

\section{DISCUSSION}

The precision, or experimental uncertainty, in the particle volume fraction measurements can be estimated from the standard deviation of the individual measurements in the middle zone, where the volume fraction is constant. These standard deviations of volume fraction are 0.002 for the radiographic measurements in Figures 2, $0.015-0.02$ for the optical microscopy measurements in Figures 5 and 6, and 0.028 for the electron microscopy measurements on the small silica particles. The corresponding uncertainties on the mean of 10 volume fraction measurements in the middle zone can be computed from those standard deviations to be \pm $0.0015, \pm 0.01-0.015$, and \pm 0.02 , respectively. Considering those uncertainties, the mean particle volume fractions measured in the middle zones of each material (values listed in the Results section) agree with values computed from weights and densities of the particle and epoxy phases. That agreement is expected, if the concentration measurements are accurate, because the computed volume fraction is the initial condition for the concentration profile prior to sedimentation and because the middle zone is unaffected by sedimentation in our experiments.

It should be noted that the accuracy of the volume fractions from microscopy refers to results uncorrected by the finite-slice-thickness term in Equation 5. Including that term would have reduced the average volume fraction in the middle zone of Figures 5 and 6 by 0.08 Volume fraction, causing a statistically significant disagreement with the computed volume fractions. It is therefore concluded that the finite thickness correction is not appropriate for volume fraction measurements of the solid and hollow glass particles by the confocal fluorescence microscopy technique. That finding cannot be explained. In contrast, the particle concentration results derived from scanning electron micrographs require no finite-thickness correction since they image the flat, polished surface of the material, instead of a section having finite thickness.

The asymmetry in the particle concentration profiles shown in Figures 5 and 6 is consistent, at least qualitatively, with theory for sedimentation (or flotation) of particles. The experimentally observed asymmetry is discerned by a gradient in concentration that is larger in absolute value (steeper slope) at the transition between the clear zone and the middle zone than at the transition between the middle zone and the maximum packed zone. In other words, the jump in concentration between the clear zone and the middle zone appears to be more abrupt than the jump in concentration between the middle zone and the maximum packed zone. That asymmetry was observed for the case of sedimenting particles in Figure 5, as well as floating particles in Figure 6. The asymmetry is consistent with the theory of gravitationally driven particle sedimentation, derived for particles large enough that hydrodynamic forces dominate over interparticle and Brownian forces $3,4,19$. For the case of a fluid having an initially uniform 
concentration of single-size (monodisperse) particles, theory shows that the rise in concentration from the middle zone having the initial concentration to the maximum packed zone can never be abrupt unless the initial concentration prior to sedimentation is either very close to the concentration in the clear zone, 0 , or very close to the concentration in the maximum packed zone. That restriction, explained in the next paragraph, is a consequence of a mass balance of particles at the interface between the middle and packed zones. In contrast, theory shows that the rise in concentration from the clear zone to the middle zone can always be abrupt (a discontinuous step), regardless of the initial concentration. Therefore, sedimentation theory for monodisperse particles predicts that the jump in concentration from the clear to the middle zone can be more abrupt than the jump from the middle to the maximum packed zone, qualitatively consistent with the results in Figures 5 and 6 . One complication in this argument is the experimental observation that even the transition between the clear and middle zones is not perfectly abrupt (Figures 5 and 6). This is believed to be a consequence of polydispersity in particle size, causing a distribution in sedimentation velocities at any given concentration. We believe, however, that the sedimentation behavior of monodisperse suspensions, that has been analyzed theoretically, is the underlying cause for the experimentally observed asymmetry in concentration profiles.

The theory-based restriction on the shape of the concentration profile in the region between the middle and maximum packed zones will now be described more quantitatively. It has been shown $3,4,19$ that a discontinuous jump in concentration between these two zones will satisfy particle mass continuity only if the particle flux and the concentration in the middle zone satisfies a requirement illustrated in Figure 11. This shows a plot of the dimensionless particle flux $\varphi U(\varphi) / U_{S}$, which is the volume fraction $\varphi$ times the particle sedimentation velocity $U$ normalized by the Stokes velocity (the sedimentation velocity for infinitely dilute particles) $U_{S}$. The so-called hindered settling function $U(\varphi) / U_{S}$ employed to make the plot in Figure 11 follows a form applicable to non-colloidal particles having size comparable to the solid and hollow glass spheres employed in this work

$$
U(\varphi) / U_{0}=(1-\varphi)^{n}
$$

where typical values of $\mathrm{n}$ are 4.6 and 5.13,19-21. The latter value was used to prepare Figure 11 , and the former value would give a curve having similar shape. The requirement for particle flux continuity at a discontinuous interface (i.e., where the particle concentration exhibits a discontinuity) between the middle and packed zones is that the straight line connecting the point representing the middle zone (having abscissa $\varphi_{0}$ and ordinate $\varphi_{0} \mathrm{U}\left(\varphi_{0}\right) / \mathrm{U}_{\mathrm{S}}$ ) and the point representing the packed zone (having coordinates $\varphi_{m}$ and 0 ) must have an ordinate value that is never higher than the ordinate value of the flux curve $\varphi U(\varphi) / U_{S}$. (The slope of that straight line is the velocity by which the discontinuity propagates through the sedimenting fluid.) The solid line in Figure 11 illustrates a middle zone (initial) volume fraction of 0.08 that is consistent with a discontinuous jump to the packed zone concentration of 0.64 , and the dotted line illustrates a middle zone concentration of 0.2 that could not satisfy particle continuity at a sharp interface. The values of initial (middle zone) concentration that would allow a discontinuous jump in concentration between the middle and packed zones has been determined numerically for the hindered settling function in Equation 6. For $n$ of 4.6 or 5.1, a discontinuous jump is not allowed if the initial particle volume fraction is in the range 0.15 to 0.50 and 0.09 to 0.53 , respectively. (An alternative form of the hindered settling function, Equation 2.1 in a review by Davis and Acrivos ${ }^{20}$, gave a similar result for the excluded range, 0.11 to 0.55 .) The initial volume fractions 
for the experimental cases shown in Figure 5, 0.49, and Figure 6, 0.48 and 0.35, are in the disallowed range for all three hindered settling functions, so a discontinuous jump from the middle zone to the packed zone would not be allowed for the case of monodisperse particles. It should be recalled that the same theory allows a discontinous jump from the clear zone to the middle zone for all values of the initial concentration. (That is intuitively reasonable, since monodisperse particles at the top of the middle zone, all moving at the same velocity should produce a sharp boundary between clear and middle zones ${ }^{19}$.) It is believed that the physics captured in this theory for monodisperse particles causes the experimentally observed concentration jump to be less abrupt between the middle and packed zones than between the clear and middle zones.

\section{CONCLUSIONS}

Filler particle concentration profiles in polymers have been measured by X-ray beam attenuation and microscopy techniques. Confocal scanning optical microscopy was employed for particles larger than $10 \mu \mathrm{m}$, and beam attenuation or electron microcopy was employed for smaller particles. The spatial resolution of the attenuation-derived concentration measurements is controlled mainly by the effective diameter of the beam. That is influenced by several characteristics of the apparatus, such as the sizes of collimators on the incident beam and on the detector, which makes the resolution somewhat uncertain. The spatial resolution of the microscopically-derived concentration measurements is easier to define, since it depends simply on the dimensions of a counting area. In our experiments on $40 \mu \mathrm{m}$ particles, measurements from images $320 \mu \mathrm{m}$ long (in the gradient direction) produced concentration profiles that agreed with expectations. For example, an asymmetry expected in profiles produced by sedimentation was observed by the microscopic technique. Near a specimen boundary, local concentration determined by the beam-attenuation method is prone to an artifact caused by the finite-diameter beam straddling the boundary. This artifact is important for determining particle concentration gradients because the gradients are often most severe near boundaries. Although most of the microscopic results were obtained on particles having sizes of 10 's of microns, local concentration of the smaller particles used in microelectronic encapsulants appeared to be measurable by employing electron micrographs instead of optical micrographs.

\section{ACKNOWLEDGEMENTS}

S. Stricker and M. Folsom of the University of New Mexico made available a laser confocal microscope and provided valuable guidance. J. Schroeder, B. McKenzie, and A. Antolak of Sandia National Laboratories performed the particle size measurements, scanning electron microscopy, and X-ray Tomographic Microscopy. C. Romero, L. Mondy, R. Rao, and A. Sun of Sandia advised the authors on hindered settling functions.

Sandia is a multiprogram laboratory operated by Sandia Corporation, a Lockheed Martin Company, for the United States Department of Energy under Contract DE-AC04-94AL85000. 
Table I

Reinforcing Particles

\begin{tabular}{|c|c|c|c|c|c|c|}
\hline Identifier & $\begin{array}{c}\text { Nominal } \\
\text { Compositon }\end{array}$ & $\begin{array}{c}\text { Particle } \\
\text { Density } \\
(\mathrm{g} / \mathrm{cc})\end{array}$ & \multicolumn{3}{|c|}{ Size Distribution Range } & \multirow{2}{*}{$\begin{array}{c}\text { DSV } \\
(\mu \mathbf{m})\end{array}$} \\
\hline & & & $\mathbf{D}_{\mathbf{1 0}}$ & $\mathrm{D}_{\mathbf{5 0}}$ & $\mathbf{D}_{\mathbf{9 0}}$ & \\
\hline Alumina & $\mathrm{Al}_{2} \mathrm{O}_{3}$ & 3.92 & 2 & 12 & 34 & \\
\hline Solid glass & $\mathrm{A}-$ glass & 2.45 & 28 & 40 & 56 & 38 \\
\hline Hollow glass & Borosilicate glass & 0.32 & 24 & 41 & 62 & 37 \\
\hline Silica & $\mathrm{SiO}_{2}$ & 2.21 & 0.6 & 2.2 & 4.7 & \\
\hline
\end{tabular}




\section{REFERENCES}

1. M. G. Pecht, L. T. Nguyen, and E. B. Hakim, Plastic Encapsulated Microelectronics (WileyInterscience, New York, 1995).

2. T. Driscoll, P. Li, G. Lehmann, and E. Cotts, in Electronic Packaging Materials Science IV, edited by S. Groothuis, P. Ho, K. Ishida and T. Wu (Mater. Res. Soc. Symp. Proc., 1997), Vol. 445, p. 69.

3. W. B. Russel, D. A. Saville, and W. R. Schowalter, Colloidal Dispersions (Cambridge University Press, Cambridge, 1989).

4. F. M. Auzerais, R. Jackson, and W. B. Russel, Journal of Fluid Mechanics 195, 437 (1988).

5. A. P. Shapiro and R. F. Probstein, Physical Review Letters 68, 1422 (1992).

6. D. Leighton and A. Acrivos, Journal of Fluid Mechanics 181, 415 (1987).

7. T. D. Papathanasiou, International Polymer Processing 11, 275 (1996).

8. D. Adolf and R. Chambers, Polymer 38, 5481 (1997).

9. J. A. Sayre, R. A. Assink, and R. R. Lagasse, Polymer 22, 87 (1981).

10. J. L. Thomason and A. Knoester, Journal of Materials Science Letters 9, 258 (1990).

11. D. L. Gard, in Methods in Cell Biology (Academic Press, 1993), Vol. 38, p. 241.

12. A. E. Ribbe, Trends in Polymer Science 5, 333 (1997).

13. C. J. R. Sheppard and D. M. Shotton, Confocal Laser Scanning Microscopy (Bios Scientific Publishers, Ltd., Oxford, 1997).

14. J. C. Russ, Practical Stereology (Plenum Press, New York, 1986).

15. T. Allen, Particle Size Measurement (Chapman and Hall, London, 1990).

16. A. J. Antolak and R. R. Lagasse, unpublished work, 1997

17. J. H. Kinney and M. C. Nichols, Annual Reviews in Materials Science 22, 121 (1992).

18. Y. Nakamura, S. Uenishi, T. Kunishi, K. Miki, H. Tabata, K. Kuwada, H. Suzuki, and T. Matsumoto, IEEE Transactions on Components, Hybrids, and Manufacturing Technology CHMT-12, 502 (1987).

19. G. B. Wallis, One-dimensional Two-phase Flow (McGraw-Hill, New York, 1969).

20. R. H. Davis and A. Acrivos, Annual Review of Fluid Mechanics 17, 91 (1985).

21. J. Garside and M. Al-Dibouni, Industrial and Engineering Chemistry, Process Design and Development 16 (1977). 


\section{Figure captions}

Figure 1. Schematic representation of particle concentration profiles produced by sedimentation. (a) Profile produced by uniform size particles having sedimentation velocity independent of local concentration (see text.) The solid profile represents a shorter sedimentation time than the dashed profile. (b) Profile produced by more realistic, polydisperse particles whose sedimentation velocity does depend on local concentration. In this case the profile has sigmoidal steps in concentration instead of discontinuities.

Figure 2. Particle concentration profiles measured by radiography near the bottom surface for two epoxy specimens filled with the same alumina. Increasing abscissa corresponds to increasing distance from the specimen bottom. The epoxy resin represented in (a) cured more slowly and therefore allowed more time for particle sedimentation than that represented in (b). The drop in concentration near the left end of the plot is an artifact (see text.)

Figure 3. (a) Reflected light optical micrograph of the epoxy filled with the hollow glass particles. This does not clearly delineate the particle outlines. The white bar represents $90 \mu \mathrm{m}$. (b) Confocal laser fluorescence micrograph of the same material, showing clearly delineated particle outlines. The white bar represents $50 \mu \mathrm{m}$. Using images like that in (b), particle volume fraction was determined within a rectangle $320 \mu \mathrm{m}$ high in the direction of the gradient and 420 $\mu \mathrm{m}$ wide.

Figure 4. Dependence of particle volume fraction on the depth below the polished surface of the microscopy specimen. The ordinate is the increment in volume fraction from the value at the surface. Volume fractions were taken to be equal to area fractions derived from confocal micrographs, without the correction shown in Equation 5 (see later text.) Closed symbols refer to the solid glass particles and open symbols to the hollow glass particles.

Figure 5. Particle concentration profiles for epoxy filled with the solid glass particles. The bottom of the specimen corresponds to 0 on the abscissa and its height was $14 \mathrm{~mm}$. The horizontal dashed line shows the average particle volume fraction of $49 \mathrm{~V} \%$ computed from the weights and densities of the constituents. The particle volume fractions do not include the correction to the area fraction given in Equation 5.

Figure 6. Particle concentration profiles for the same epoxy filled with $48 \mathrm{~V} \%$ (filled symbols) and $35 \mathrm{~V} \%$ (open symbols) of the hollow glass particles. The bottom of each specimen corresponds to 0 on the abscissa and the height of both specimens was $13 \mathrm{~mm}$. The horizontal dashed lines shows the average particle volume fractions computed from weights and densities of the constituents. The particle volume fractions have not been corrected using Equation 5 .

Figure 7. Confocal laser fluorescence micrograph of an epoxy modified by CTBN elastomeric particles and filled with 0.48 volume fraction of the hollow glass particles. The elastomeric particles are distinguished because they are brighter, due to higher concentration of fluorescent dye, than the glass particles. Image analysis produced a concentration of glass particles agreeing with the expected value (see text.)

Figure 8. Schematic representation of two locations in a test specimen at which micrographs in Figure 9 were obtained. The two spherical electrodes were encapsulated by an epoxy filled with the hollow glass particles. The upper and lower rectangles represent locations near the electrode- 
epoxy interface that are shown in Figures 9(a) and (b), respectively. During the encapsulation, the particles floated vertically, opposite to the direction of gravity.

Figure 9. Confocal micrographs illustrating qualitatively the effect of particle flotation on the concentration profile near the surface of the spherical electrodes illustrated in Figure 8. Figure 9 (a) and (b) were obtained near the upper and lower rectangular areas in the previous figure. The hollow particles floated oppositely to the white arrow, which signifies gravity. The upper region, Figure 9(a), exhibits a layer of depleted particle concentration near the electrode surface, caused by flotation. This depletion zone is absent in the lower region, Figure 9(b).

Figure 10. Scanning electron microscopy backscatter image of the small-size silica particles dispersed in epoxy (without the toughening additive). Analysis of several of these images produced a particle volume fraction agreeing to within $2 \%$ with the known concentration. The white bar represents $2 \mu \mathrm{m}$.

Figure 11. A plot of the dimensionless sedimentation flux given by Equation 6 with $n=5.1$. The lines connect the point corresponding to the maximum packed zone at volume fraction $\varphi=0.64$ with two points on the flux curve corresponding to different initial particle concentrations, $\varphi=0.08$ (solid) and 0.2 (dashed.) The former case allows a discontinuous jump in particle concentration from the middle zone to the maximum packed zone but the latter case does not, for reasons explained in the text. This plot leads to a reasonable explanation for the experimental finding that the rise in particle concentration is more abrupt between the clear and middle zones than between the middle and packed zones. 
Figure 1(a)

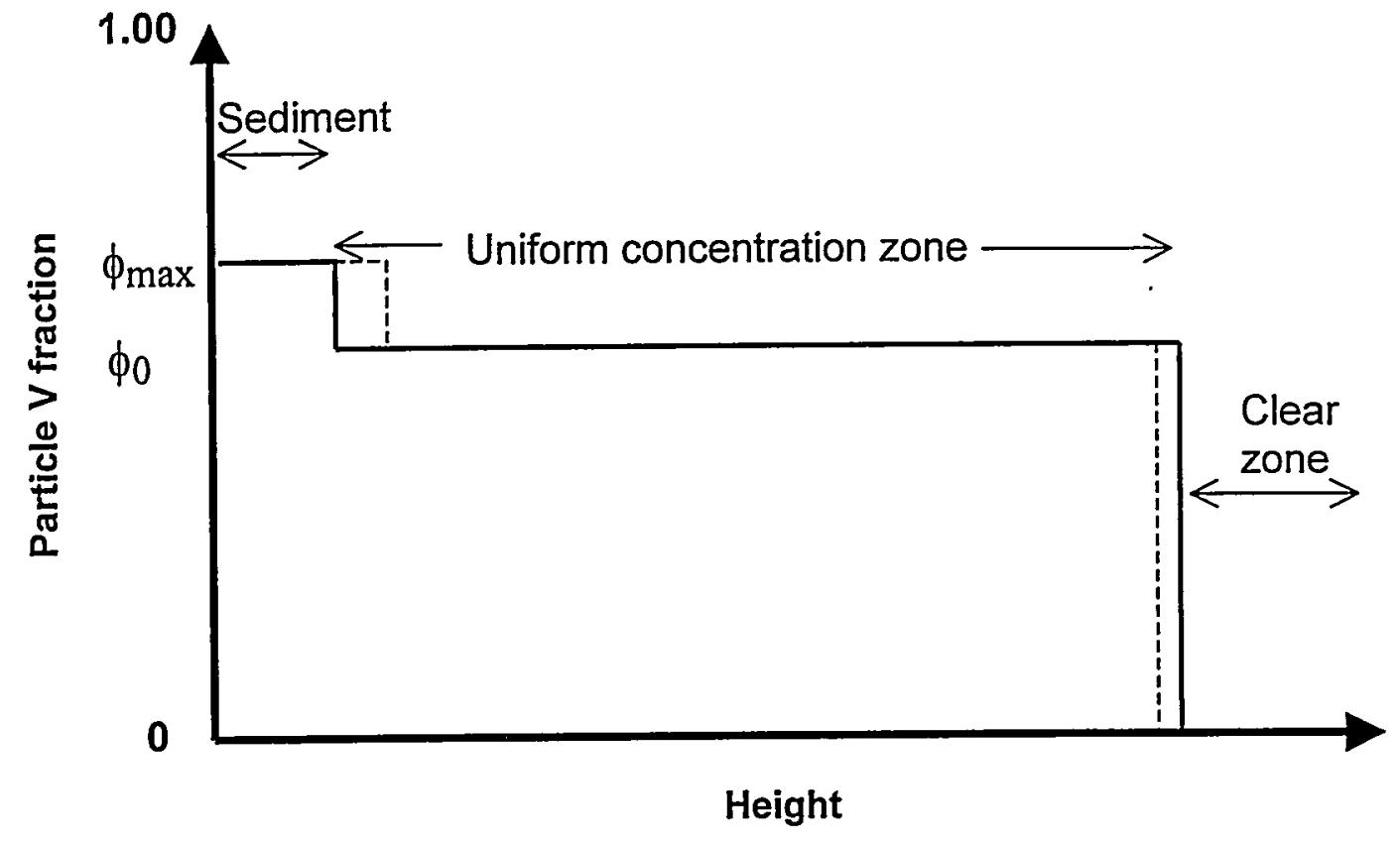

Figure 1(b)

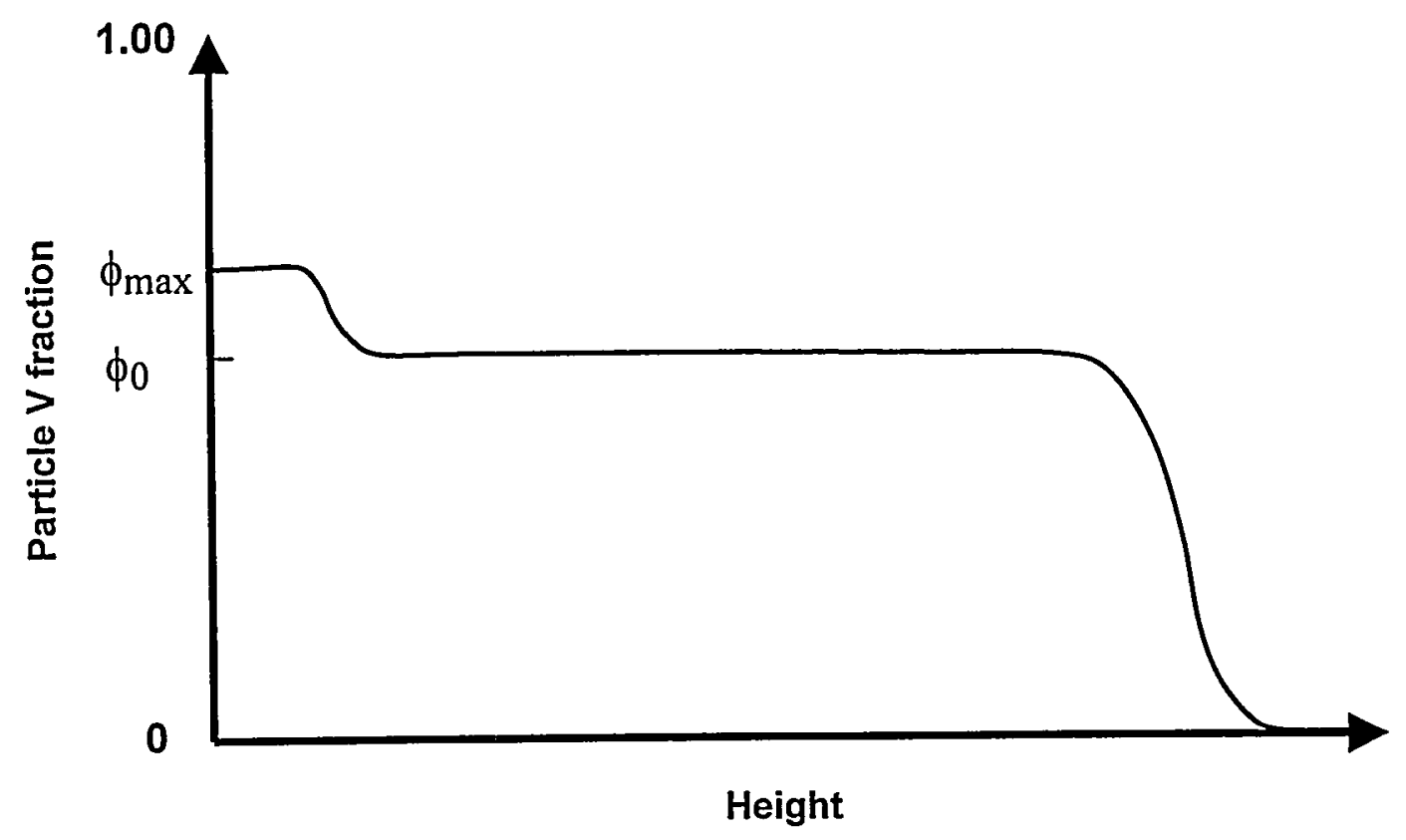


Figure 2a

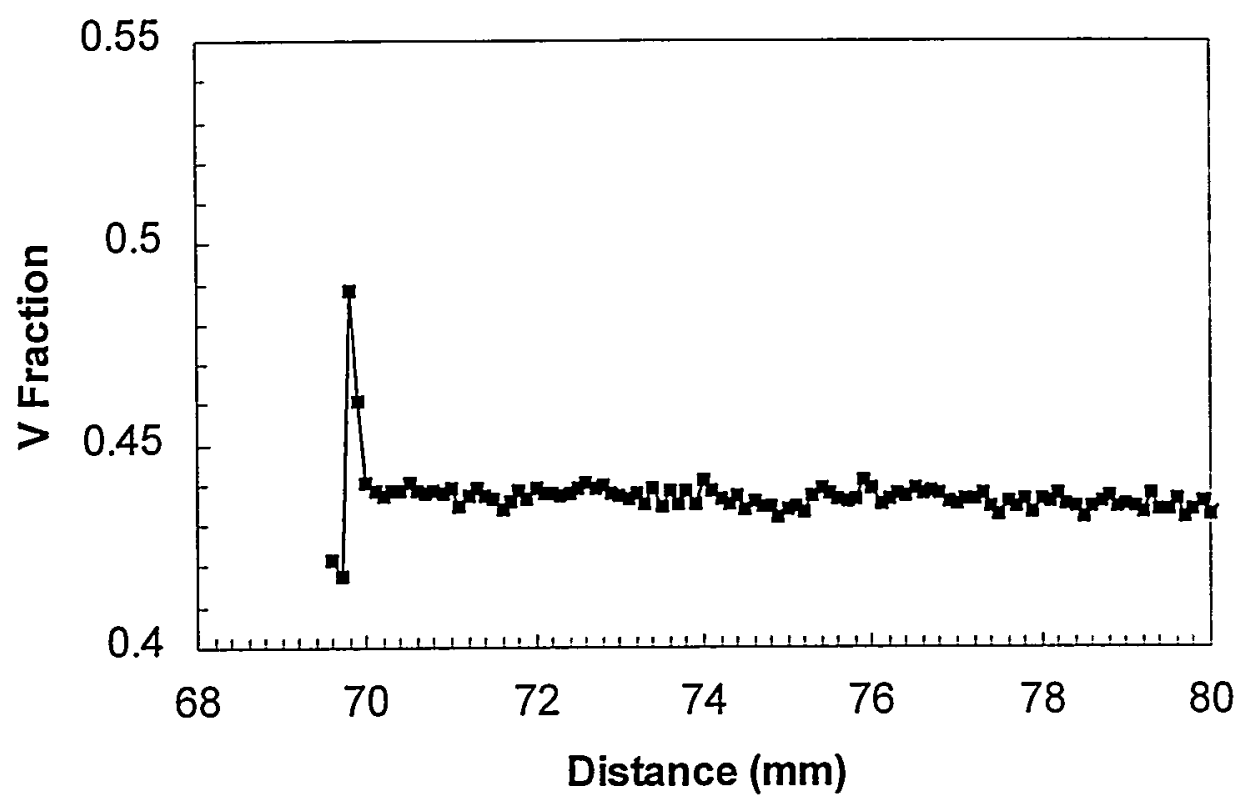

Figure 2(b)

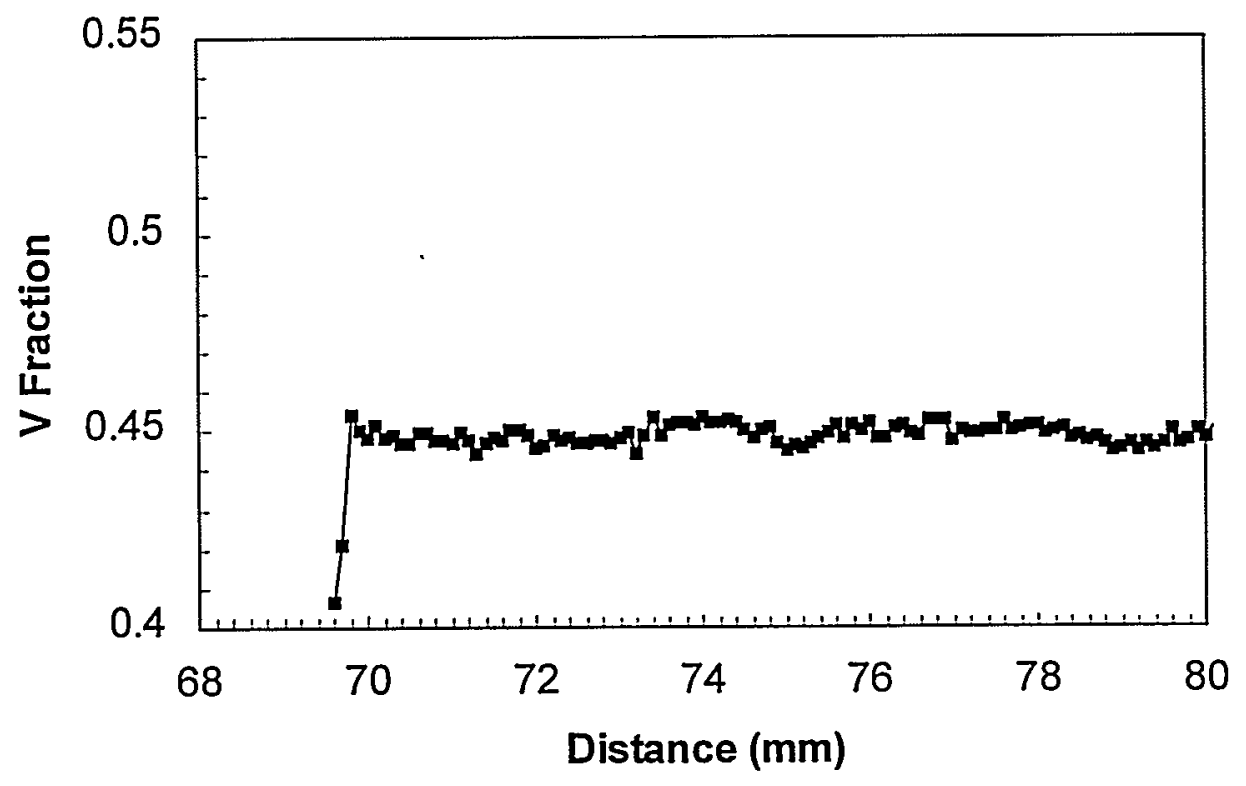


Figure $3 a$

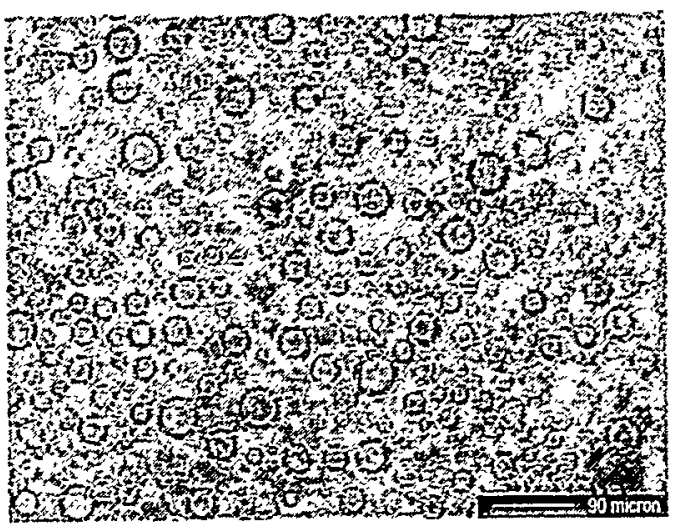

Figure $3 b$

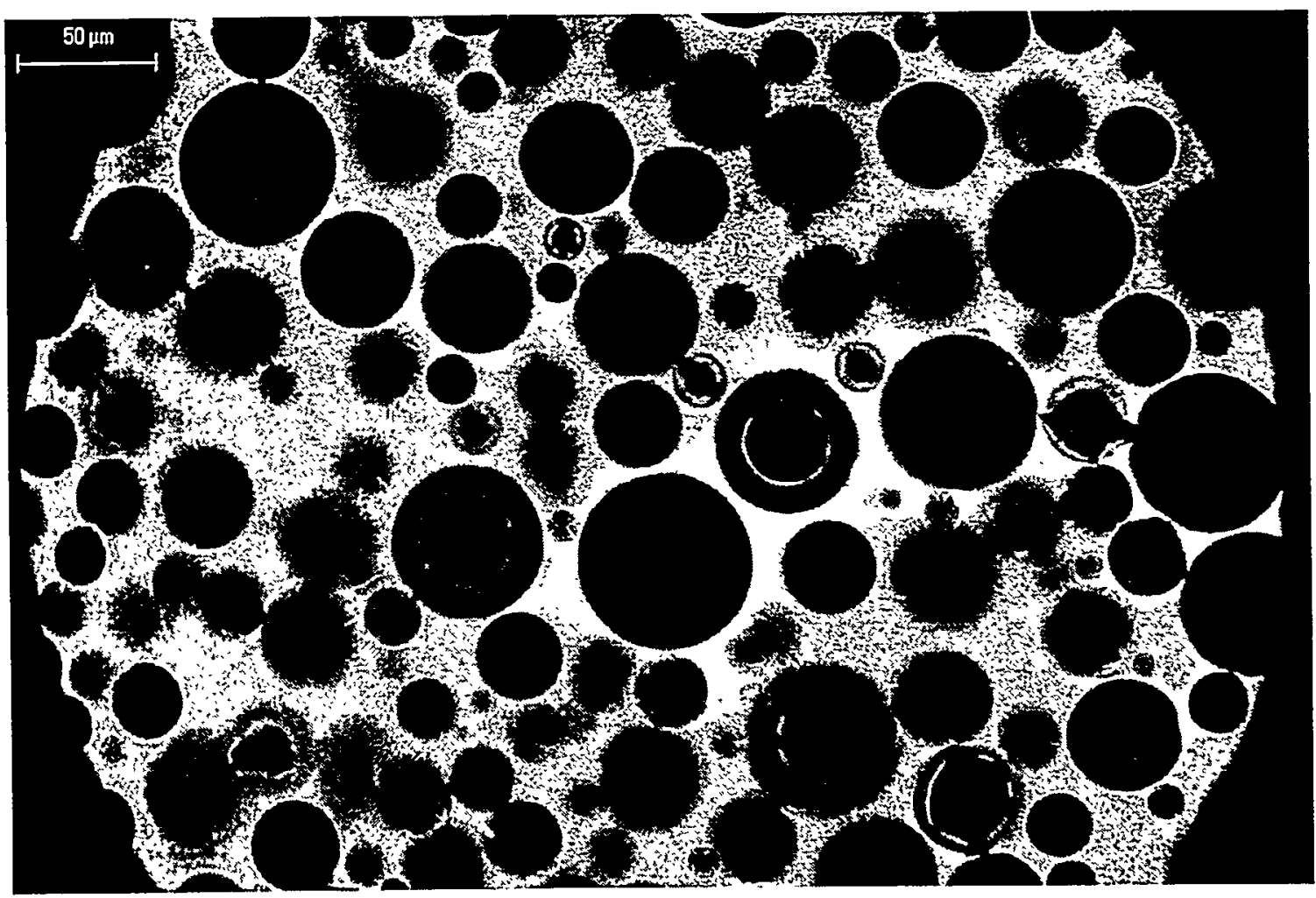




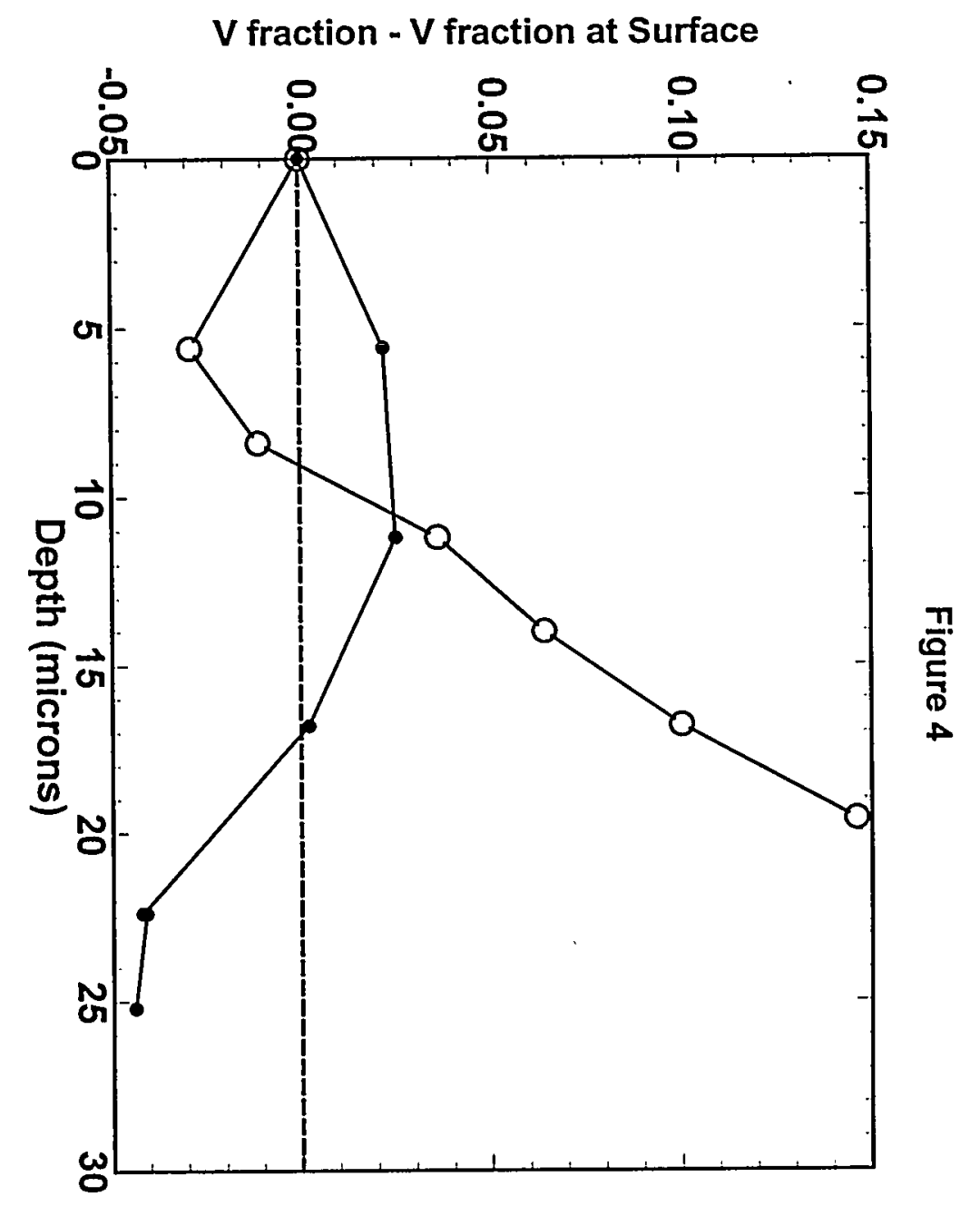


Figure 5

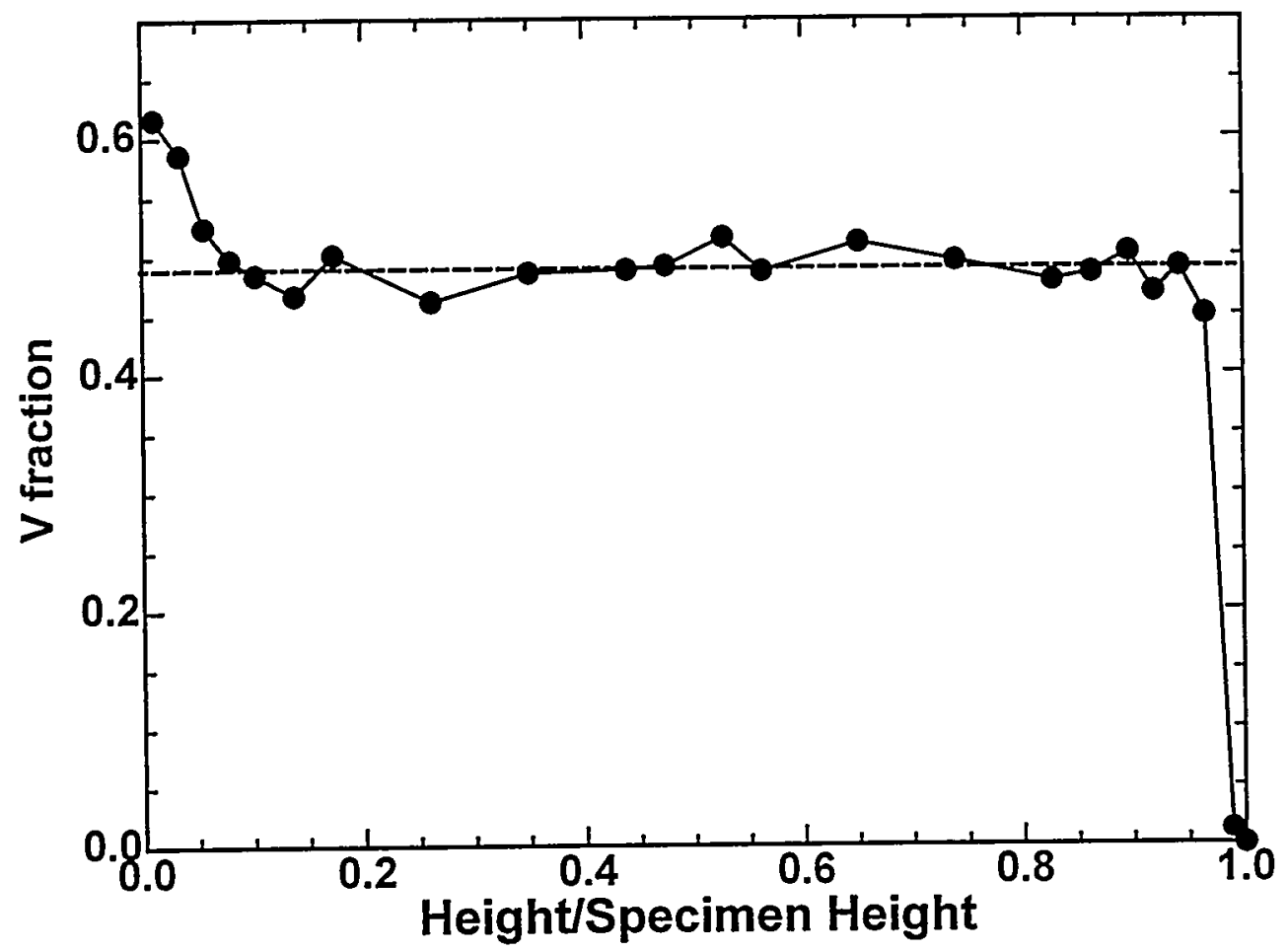


Figure 6

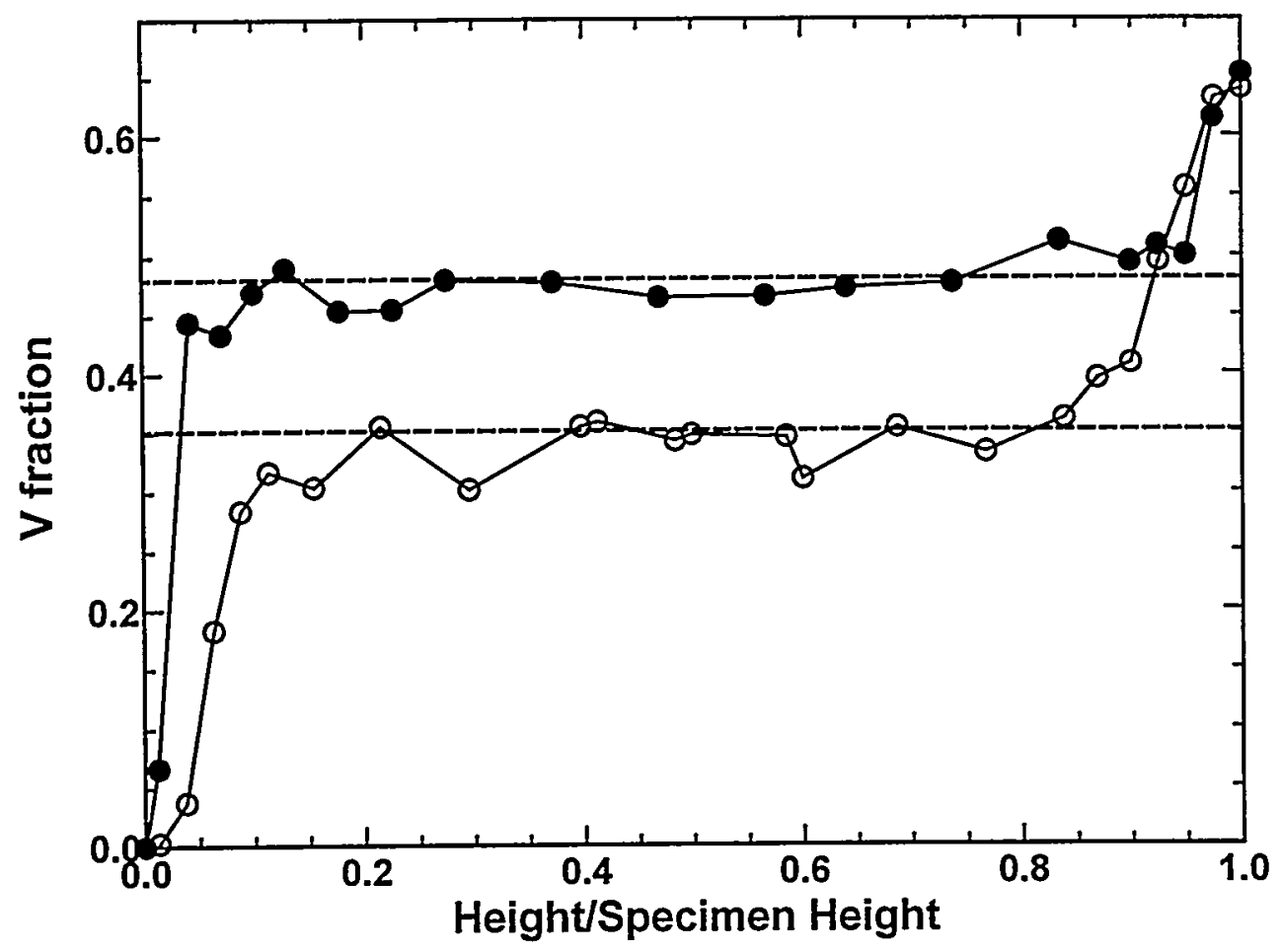

Figure 7

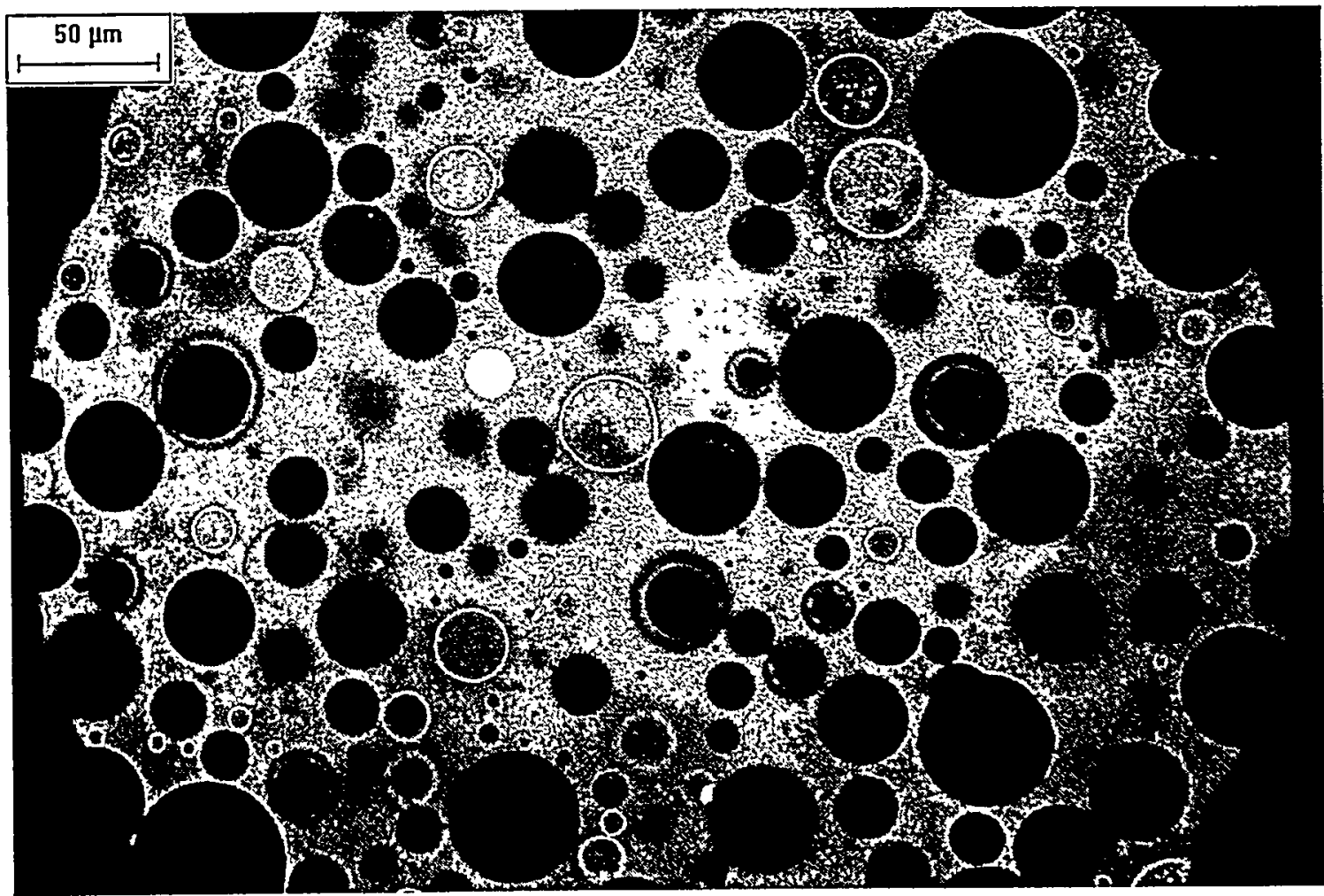


Figure 8

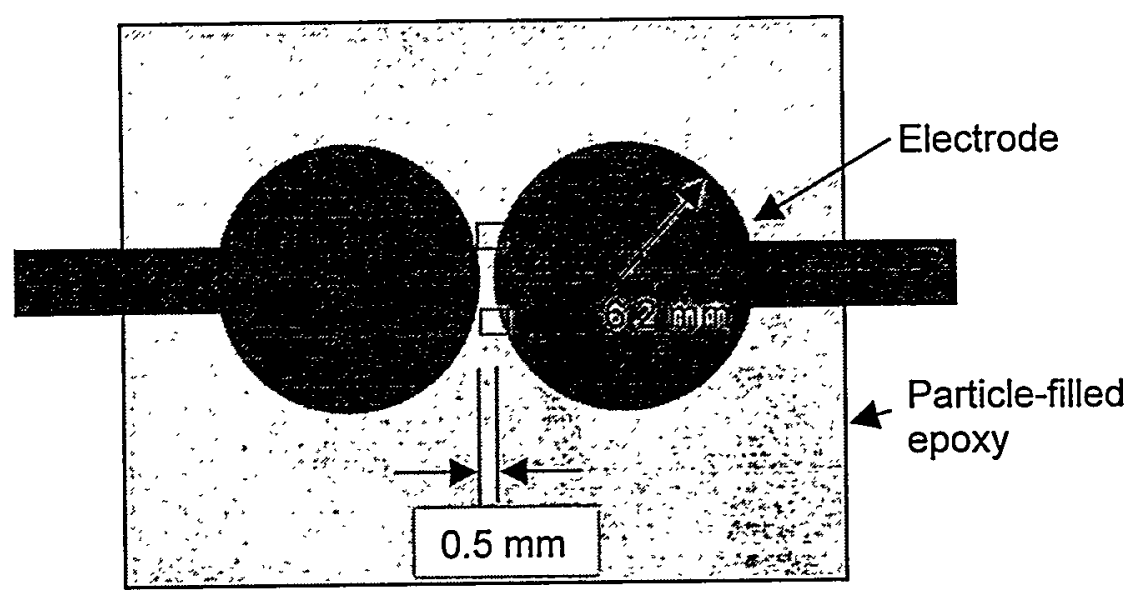


Figure 9(a)

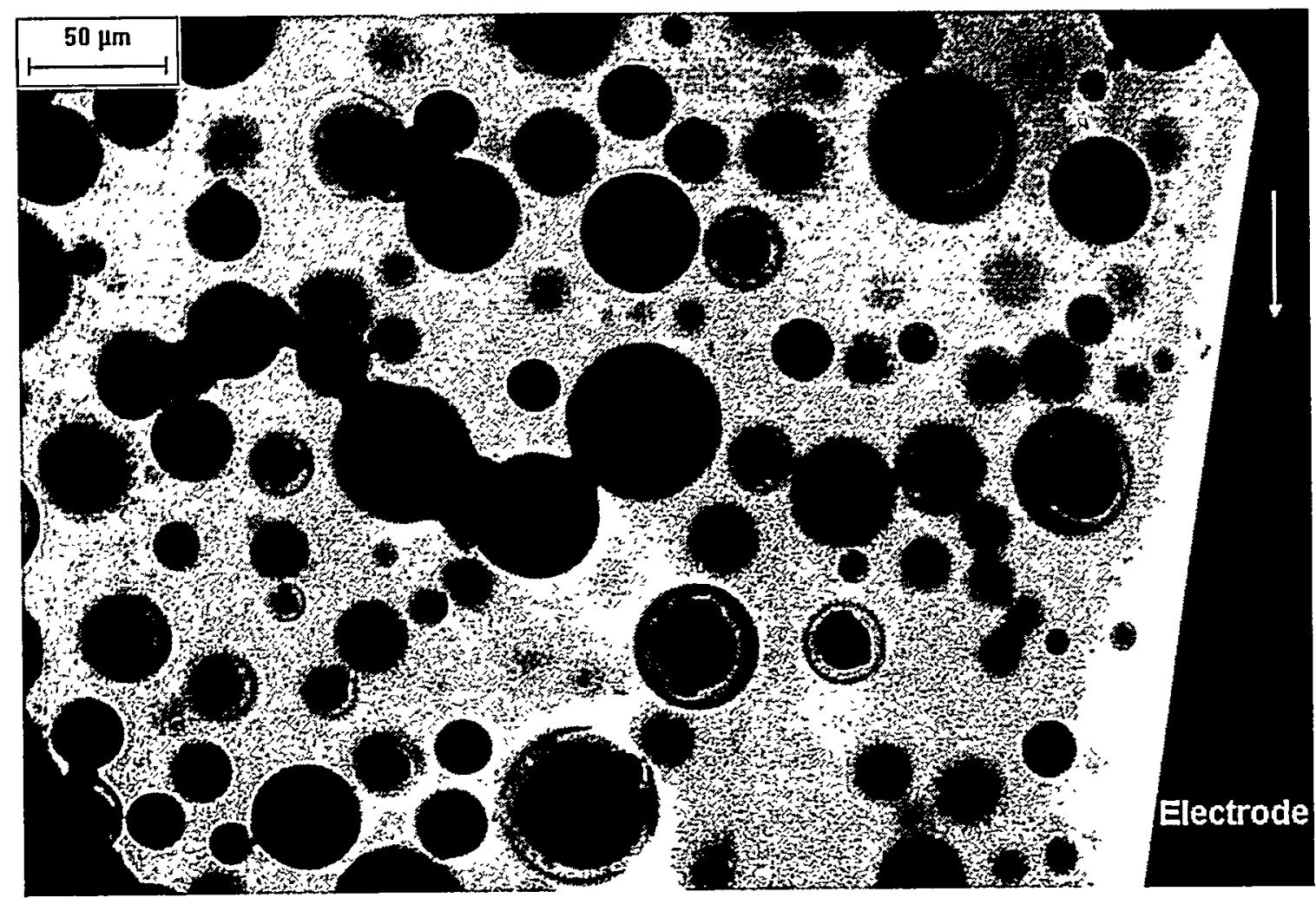

Figure $9(b)$

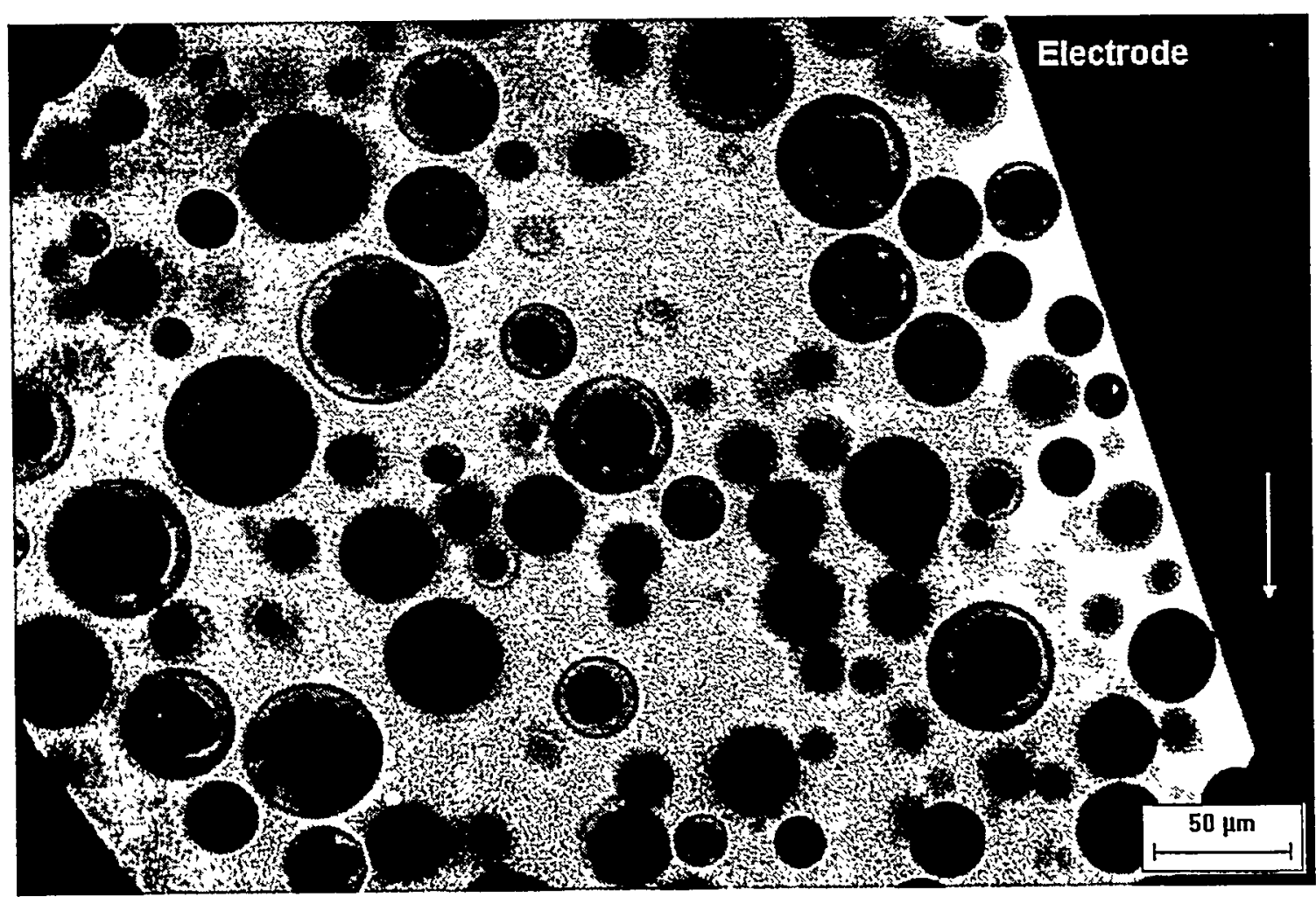


Figure 10

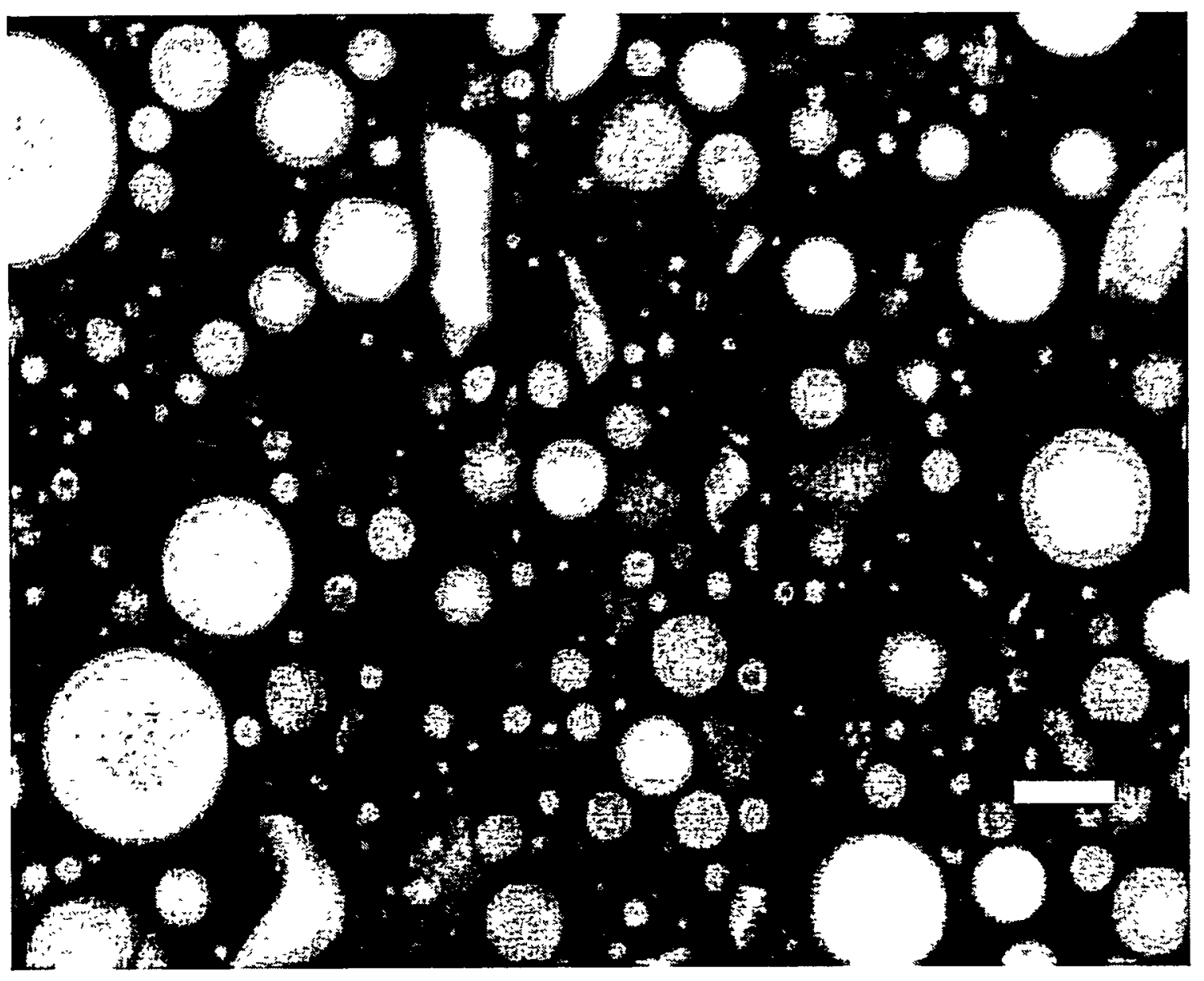


Figure 11

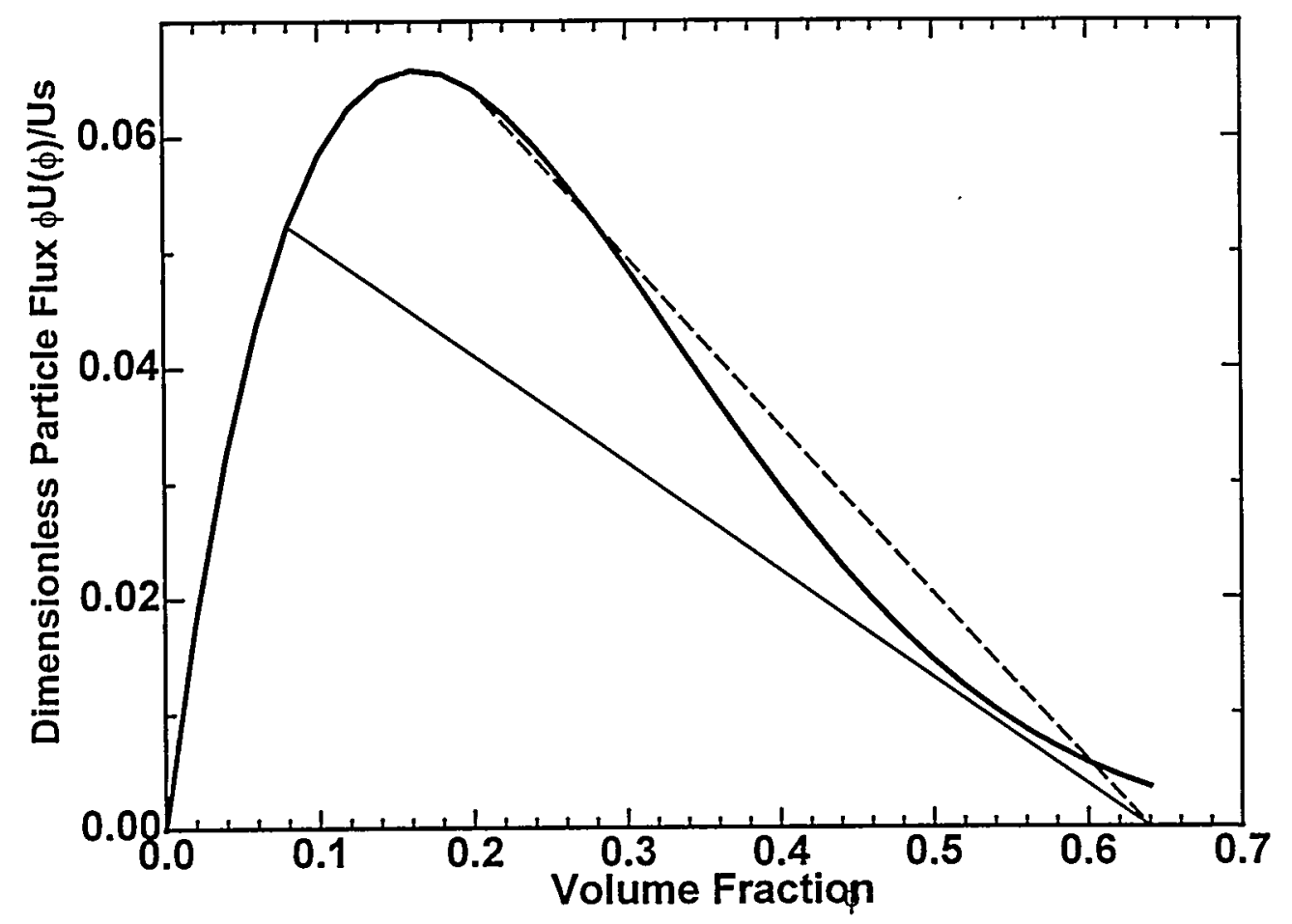

